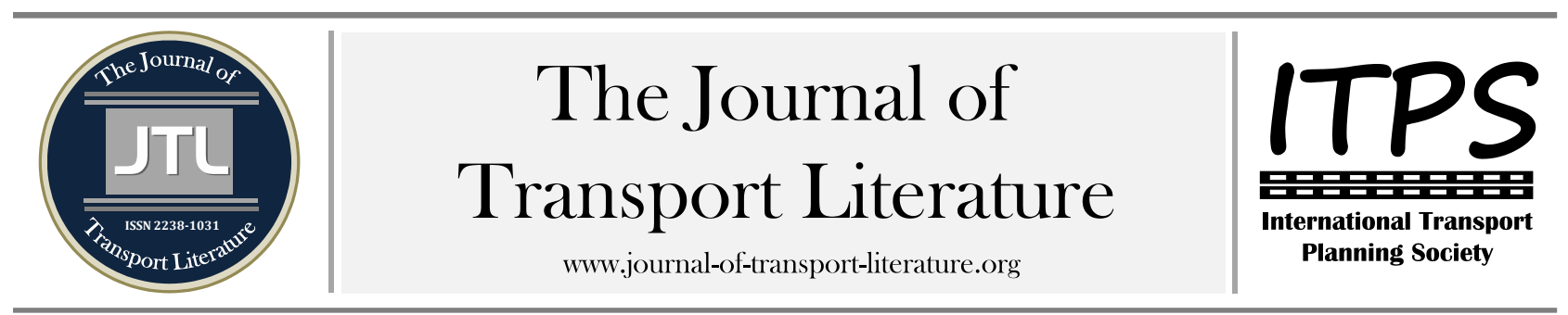

\title{
Transporte coletivo: vibração de corpo-inteiro e conforto de passageiros, motoristas e cobradores
}

Marilu Alcântara de Melo Figueiredo+; Luiz Felipe Silva; Tiago Leão Barnabé

Universidade Federal de Itajubá, Itajubá, Brasil

\section{Article Info}

Palavras-chave:

Vibração de corpo-inteiro

Motoristas

Cobradores

Passageiros

Conforto

Submitted 6 Jan 2015

received in revised form 21 Jul 2015

accepted 31 Aug 2015

Licensed under

Creative Commons

CC-BY $3.0 \mathrm{BR}$.

\section{Resumo}

A vibração de corpo-inteiro (VCI) está presente em diversos veículos, incluindo os ônibus urbanos. A exposição excessiva está associada ao risco de distúrbios na região lombar. Este estudo objetivou avaliar a exposição de condutores e passageiros à VCI. Foi empregada a ISO-2631-1997 como norma de referência para avaliação, e nos limites ocupacionais da Diretiva Europeia (2002). Observou-se superação do nível de ação, segundo o método básico, nos três eixos, tanto para o cobrador como para o motorista do ônibus. A superação do nível limite, segundo método da quarta potência, ocorreu no eixo z, para cobrador e motorista, com valores de VDV de 36,28 ms-1,75 e de 39,71 ms-1,75, respectivamente. Para a realidade do passageiro, a situação no assento frontal do veículo, o valor observado da exposição à VCI, pelo método básico, foi considerado "um pouco desconfortável", enquanto que para o sentado na seção central, foi classificada como "desconfortável". Os valores médios das magnitudes de vibração, no método básico, eixo z, encontrados nos assentos dos passageiros e dos condutores são similares, sugerindo que o amortecimento do assento destes é inadequado. Os condutores estão expostos a níveis acima dos limites recomendados, e os passageiros têm viajado em situação desconfortável.

+ Corresponding author. Universidade Federal de Itajubá. Av. BPS, 1303, Pinheirinho. 37500-903 - Itajuba, MG - Brasil.

E-mail address: marilualcantara08@gmail.com

\section{Introdução}

A exposição à Vibração de Corpo-Inteiro (VCI) pode causar desconforto e alterações nos sistemas corpóreos e pesquisas têm sido realizadas com o intuito de conhecer a reação do corpo humano frente a este agente agressor. A Portaria 1339 do Ministério da Saúde (MS) considera as vibrações como um agente de risco de natureza ocupacional (Brasil, 1999).

0 transporte público coletivo tem sido um dos meios de locomoção mais utilizados pelas pessoas e o ônibus é uma das opções de transporte mais procuradas nesta categoria. 0 conforto no interior dos ônibus para os passageiros tem sido avaliado no tocante ao ruído (Silva e Correia, 2012), contudo há limitação de análises na área dos efeitos decorrentes da vibração. 0 ambiente, representado pelo interior dos ônibus utilizados no transporte público coletivo, expõe à VCI, tanto o passageiro quanto motorista e cobrador. As patologias relacionadas à coluna vertebral, um dos desfechos decorrentes da exposição à VCI, representam razões de comprometimento das atividades laborativas, ausências ao trabalho e são os tópicos patológicos mais recorrentes na literatura. 0 passageiro pode ficar exposto desde poucos minutos até horas acomodado no interior do ônibus, submetido a estímulos vibratórios oriundos dos mecanismos e estrutura do veículo em movimento e sua interface com a qualidade do pavimento.

Este estudo tem como objetivo avaliar a exposição de motorista, cobrador e passageiro à VCI. Na seção 1 do estudo, apresentando a sua estrutura de construção, há a abordagem sobre o referencial teórico acerca do tema. Os materiais e métodos utilizados na condução do estudo estão expostos na seção 2, com os resultados e discussão apresentados na seção 3. A conclusão do estudo está contida na seção 4.

\section{Referencial teórico}

De acordo com Silva (2005), a VCI é um estímulo presente em diversas realidades de trabalho. É frequentemente encontrada na indústria de transporte como caminhões, ônibus, motocicletas e veículos em geral. Naturalmente é diferenciada a exposição à VCI de um trabalhador envolvido diretamente em seu processo de trabalho da pessoa que é exposta esporadicamente ou por poucos minutos/horas ao longo do dia. Um dos possíveis efeitos que podem ser oriundos da VCI é a sensação de desconforto e dor, frequentemente avaliada nos passageiros dos veículos. Segundo Griffin et al. (2006), a transmissão da vibração ao corpo está relacionada à postura e devido a esta razão os seus efeitos são complexos.

A resposta humana à VCI é muito complexa e tem sido usado um conjunto de normas para abordá-la. Para Griffin (1998), a norma ISO 2631-1 (1997) apresenta uma alternativa de métodos para quantificação da exposição à vibração no corpo humano em relação à saúde e conforto. Estes métodos podem avaliar a vibração em veículos e máquinas que podem interferir no conforto, na saúde e no desempenho das atividades laborais. Os códigos de severidade encontrados na referida norma dividem os limites em: a) conforto - aplicável a passageiros de veículos; b) perda de eficiência causada por fadiga relacionado à preservação da eficiência no trabalho, aplicado aos motoristas e cobradores e c) exposição sob condições 
específicas que oferecem perigo à saúde. 0 Quadro 1 ilustra os valores de aceleração em ms-2 (r.m.s) indicando a percepção do corpo humano em relação ao conforto. De acordo com Nelson e Brereton (2005) a avaliação deve se balizar com os limites estabelecidos pela Diretiva Europeia, fundamentados no valor registrado da aceleração equivalente para uma jornada de oito horas [A(8)] e do Valor da Dose de Vibração (VDV), métodos básico e da quarta potência, respectivamente, a saber: valor de ação para exposição diária: 0,5 m/s-2 A(8) ou VDV de 9,1 m/s-1,75; valor limite para exposição diária: 1,15 $\mathrm{m} / \mathrm{s}-2 \mathrm{~A}(8)$, ou VDV de $21 \mathrm{~ms}-1,75$.

Quadro 1 - valores da aceleração ms-2 (r.m.s.) em relação ao conforto.

Fonte: ISO 2631/1 (1997).

\begin{tabular}{|c|c|}
\hline Aceleração r.m.s $\left(\mathbf{m s}^{-\mathbf{2}}\right)$ & Classificação \\
\hline$<0,315$ & Confortável \\
\hline 0,315 a 0,63 & Um pouco desconfortável \\
\hline 0,80 a 1,60 & Desconfortável \\
\hline 1,25 a 2,50 & Muito desconfortável \\
\hline$>2,00$ & Extremamente desconfortável \\
\hline
\end{tabular}

\section{Material e métodos}

Trata-se de um estudo observacional, cuja amostra foi composta por um motorista do sexo masculino, 29 anos, $72 \mathrm{~kg}$, $1,74 \mathrm{~m}$; um cobrador do sexo masculino, 28 anos, $88 \mathrm{~kg}, 1,70 \mathrm{~m}$ e uma passageira, no caso um dos autores desta pesquisa, 41 anos, $59 \mathrm{~kg}, 1,64 \mathrm{~m}$, acomodada no primeiro assento e em outra medição, posicionada em assento no centro do veículo. Os assentos dos condutores possuem sistema de amortecimento e ajuste, enquanto que o dos passageiros apresenta uma configuração rígida. 0 veículo utilizado foi um ônibus dotado de chassis marca Mercedes Benz (Modelo OF1435) com carroceria Apache com capacidade para 37 passageiros, ano de fabricação 2011. Em relação ao pavimento, o trajeto escolhido para análise apresentou 6,2 km de asfalto de ida e 6,7 km de asfalto de volta; 5,2 km de calçamento de ida e 5,1 km de calçamento de volta perfazendo um total de $11,4 \mathrm{~km}$ de ida e $11,8 \mathrm{~km}$ de volta. A empresa de transporte público coletivo, fonte das amostras, está localizada em uma cidade do interior do estado de Minas Gerais. Conta em seu quadro de condutores, 168 trabalhadores (motoristas e cobradores), compreendendo 37 veículos distribuídos em 18 linhas ou trajetos. 0 procedimento de avaliação empregou um conjunto de equipamentos constituído por um medidor de vibração, marca Larson\&Davis, modelo HVM100, e acelerômetro triaxial de assento disposto entre o sujeito e o assento do veículo. 0 tempo de duração da medição correspondeu ao tempo total despendido na viagem de ida e volta. Foram utilizados os métodos básico e o da quarta potência (ISO2631,1997). Para o cálculo da avaliação da exposição do passageiro, lançou-se mão de estimativa de tempo de uso de transporte coletivo, variando de 15 min até $8 \mathrm{~h}$ por dia.

Os resultados obtidos da avaliação foram cotejados de acordo com os valores recomendados pela Diretiva Europeia, no que tange à superação dos níveis de ação e limite, além das diretrizes definidas pela ISO 2631 - 1997. Cada trecho da viagem foi considerado como uma "máquina" distinta operada pelos condutores, no cálculo da exposição geral, desde que as condições do pavimento e da carga do veículo e velocidade se alteravam. Para o cálculo da aceleração equivalente a uma jornada de oito horas, segundo o método básico, foi utilizada a Equação 1:

onde:

$$
A_{j}(8)=k \times a_{w j} \sqrt{\frac{t_{e}}{t_{0}}} m s^{-2}
$$

$\mathrm{k}$ : fator de correção de 1,4 para os eixos x, e y e de 1,0 para o eixo $\mathrm{z}$;

aw,j: valor da aceleração ponderada para o eixo j;

te: tempo de exposição em horas;

t0: tempo de referência equivalente a oito horas.

Considerando que cada trecho, em número de seis, da viagem foi tratado como uma avaliação distinta, o valor diário foi calculado de acordo com a Equação 2:

$$
A_{j}(8)=\sqrt{\sum_{i=1}^{6} A_{j i}^{2} m s^{-2}}
$$

onde:

A j,i: valor da aceleração registrado em cada trajeto.

Em referência à avaliação pelo método da quarta potência, o cálculo da exposição de acordo com valor da dose de vibração (VDV) se fez pela Equação 3:

onde:

$$
V D V_{j, i}=k \times V D V_{j}^{4} \sqrt{\frac{t_{e}}{t_{\text {med }}}} m s^{-1,75}
$$

$\mathrm{k}$ : fator de correção de 1,4 para os eixos x, e y e de 1,0 para o eixo z;

te: tempo de exposição em horas;

tmed: tempo de medição.

Do mesmo modo, em relação aos seis trechos do trajeto, foi calculado o valor total da exposição, segundo o VDV, pela Equação 4: 


$$
V D V_{j}=\sqrt[4]{\sum_{i=1}^{6} V D V_{j}^{4}} m s^{-1,75}
$$

onde:

VDVj: valor da dose de vibração no trecho j.

Este trabalho faz parte de um estudo maior intitulado "Dor lombar em condutores de ônibus: investigação da associação com a exposição à VCI". A medição foi aplicada após o cadastramento do trabalho na Plataforma Brasil e posterior aprovação pelo Comitê de Ética em Pesquisa (CEP) da Faculdade de Medicina de Itajubá, parecer consubstanciado número 400.744, data da relatoria em 19 de setembro de 2013.

\section{Resultados e discussão}

Os resultados das avaliações das exposições do motorista e do cobrador estão expostos na Tabela 1.0 valor de exposição à VCI não excedeu o nível limite para o motorista, não ocorrendo o mesmo com o cobrador. As observações sugerem que as características de amortecimento do assento do motorista podem contribuir para seu maior conforto.

Tabela 1 - Valores dos níveis de exposição à VCI, por trechos do trajeto, segundo os métodos básico e da quarta-potência, para o motorista e o cobrador.

Fonte: "com os próprios cálculos".

\begin{tabular}{|c|c|c|c|c|c|c|c|c|c|c|c|c|}
\hline \multicolumn{13}{|c|}{ Motorista } \\
\hline \multirow{2}{*}{ Trechos } & \multicolumn{3}{|c|}{ Valores da vibração $\left(\mathrm{ms}^{-2}\right)$} & \multicolumn{3}{|c|}{$\begin{array}{c}\text { Valores diários da vibração } \\
\left(\mathrm{ms}^{-2}\right)\end{array}$} & \multicolumn{3}{|c|}{$\begin{array}{c}\begin{array}{c}\text { Valores da dose da vibração } \\
\left(\mathrm{ms}^{-1,75}\right)\end{array} \\
\end{array}$} & \multicolumn{3}{|c|}{$\begin{array}{l}\text { Valores da exposição da } \\
\text { dose da vibração }\left(\mathrm{ms}^{-1,75}\right)\end{array}$} \\
\hline & $\mathrm{Ax}$ & $\mathrm{A}_{\mathrm{x}}$ & $\mathrm{A}_{z}$ & $\mathrm{Ax}_{\mathrm{x}}(8)$ & $A_{y}(8)$ & $\mathrm{A}_{z}(8)$ & $\mathrm{VDV}_{\mathrm{x}}$ & $\mathrm{VDV}_{\mathrm{y}}$ & $\mathrm{VDV}_{z}$ & $\begin{array}{l}\text { VDV } \\
\exp _{x}\end{array}$ & $\begin{array}{l}\text { VDV } \\
\text { expy }\end{array}$ & $\begin{array}{l}\text { VDV } \\
\exp z\end{array}$ \\
\hline A & 0,34 & 0,35 & 0,88 & 0,21 & 0,22 & 0,40 & 1,64 & 1,80 & 4,77 & 5,36 & 5,88 & 15,59 \\
\hline B & 0,35 & 0,34 & 0,85 & 0,22 & 0,21 & 0,38 & 3,14 & 2,88 & 7,40 & 10,26 & 9,41 & 24,18 \\
\hline C & 0,36 & 0,35 & 0,89 & 0,23 & 0,22 & 0,40 & 3,04 & 2,95 & 7,86 & 9,93 & 9,64 & 25,69 \\
\hline D & 0,37 & 0,36 & 0,98 & 0,23 & 0,23 & 0,44 & 3,04 & 2,88 & 8,20 & 9,93 & 9,41 & 26,80 \\
\hline$E$ & 0,35 & 0,32 & 0,32 & 0,22 & 0,20 & 0,14 & 2,97 & 2,75 & 7,94 & 9,71 & 8,99 & 25,95 \\
\hline $\mathrm{F}$ & 0,29 & 0,27 & 0,27 & 0,18 & 0,17 & 0,12 & 2,60 & 2,38 & 7,87 & 8,50 & 7,78 & 25,72 \\
\hline $\mathrm{G}$ & 0,28 & 0,26 & 0,26 & 0,18 & 0,16 & 0,12 & 2,76 & 2,41 & 6,80 & 9,02 & 7,88 & 22,22 \\
\hline Somatório & & & & 0,56 & 0,54 & 0,84 & & & & 15,11 & 14,10 & 39,71 \\
\hline \multicolumn{13}{|c|}{ Cobrador } \\
\hline \multirow{2}{*}{ Trechos } & \multicolumn{3}{|c|}{ Valores da vibração $\left(\mathrm{ms}^{-2}\right)$} & \multicolumn{3}{|c|}{$\begin{array}{l}\text { Valores diários da vibração } \\
\left(\mathrm{ms}^{-2}\right)\end{array}$} & \multicolumn{3}{|c|}{$\begin{array}{c}\text { Valores da dose da vibração } \\
\left(\mathrm{ms}^{-1,75}\right)\end{array}$} & \multicolumn{3}{|c|}{$\begin{array}{l}\text { Valores da exposição da dose } \\
\text { da vibração }\left(\mathrm{ms}^{-1,75}\right)\end{array}$} \\
\hline & $\mathrm{Ax}_{\mathrm{x}}$ & $\mathrm{Ax}$ & $\mathrm{A}_{z}$ & $A_{x}(8)$ & $A_{y}(8)$ & $\mathrm{A}_{z}(8)$ & $\mathrm{VDV}_{\mathrm{x}}$ & $\mathrm{VDV}_{\mathrm{y}}$ & $\mathrm{VDV}_{z}$ & $\begin{array}{l}\text { VDV } \\
\text { exps }\end{array}$ & $\begin{array}{l}\text { VDV } \\
\text { expoy }\end{array}$ & $\begin{array}{l}\text { VDV } \\
\text { exp.z }\end{array}$ \\
\hline A & 0,59 & 0,12 & 0,2 & 0,37 & 0,08 & 0,09 & 0,54 & 1,08 & 0,97 & 1,70 & 3,41 & 3,06 \\
\hline B & 0,27 & 0,44 & 0,74 & 0,17 & 0,28 & 0,33 & 2,32 & 3,94 & 6,34 & 7,32 & 12,43 & 20,00 \\
\hline C & 0,34 & 0,59 & 0,92 & 0,21 & 0,37 & 0,42 & 2,91 & 5,18 & 7,80 & 9,18 & 16,34 & 24,60 \\
\hline D & 0,32 & 0,55 & 0,93 & 0,20 & 0,35 & 0,42 & 2,87 & 4,81 & 7,99 & 9,05 & 15,17 & 25,20 \\
\hline $\mathrm{E}$ & 0,29 & 0,51 & 0,76 & 0,18 & 0,32 & 0,34 & 2,64 & 4,59 & 6,75 & 8,33 & 14,48 & 21,29 \\
\hline F & 0,27 & 0,35 & 0,65 & 0,17 & 0,22 & 0,29 & 2,42 & 3,24 & 5,94 & 7,63 & 10,22 & 18,74 \\
\hline $\mathrm{G}$ & 0,33 & 0,59 & 0,99 & 0,21 & 0,37 & 0,45 & 2,88 & 5,16 & 8,32 & 9,08 & 16,28 & 26,24 \\
\hline Somatório & & & & 0,60 & 0,80 & 0,93 & & & & 13,34 & 22,86 & 36,28 \\
\hline
\end{tabular}

Para o cobrador, conforme verificado na Tabela 1, houve superação do nível de ação para o método básico nos três eixos, sendo considerado o valor da exposição diária no eixo z de 0,93m/s-2. Ainda em relação a este condutor, pode ser observada a superação dos níveis limite para a quarta potência no tocante aos eixos y e z, sendo este último o valor da exposição.

No que tange ao motorista, observou-se que, para o método básico, o eixo z é considerado o valor de exposição, superando o nível de ação, assim como os demais eixos ortogonais. Novamente a superação do nível de ação foi constatada, conforme o método da quarta potência, registrando ainda superação do limite para o eixo $\mathrm{z}$, sendo, portanto, considerado 0 valor de exposição.

No que tange ao passageiro sentado no assento localizado na seção frontal do ônibus, pode ser verificada a estimativa de exposição, a partir de valores hipotéticos de tempo de uso do veículo no cotidiano, variando de 15 min a 8 h. Os resultados alcançados pela avaliação, conforme o método da quarta potência, podem ser observados na Figura 1. 


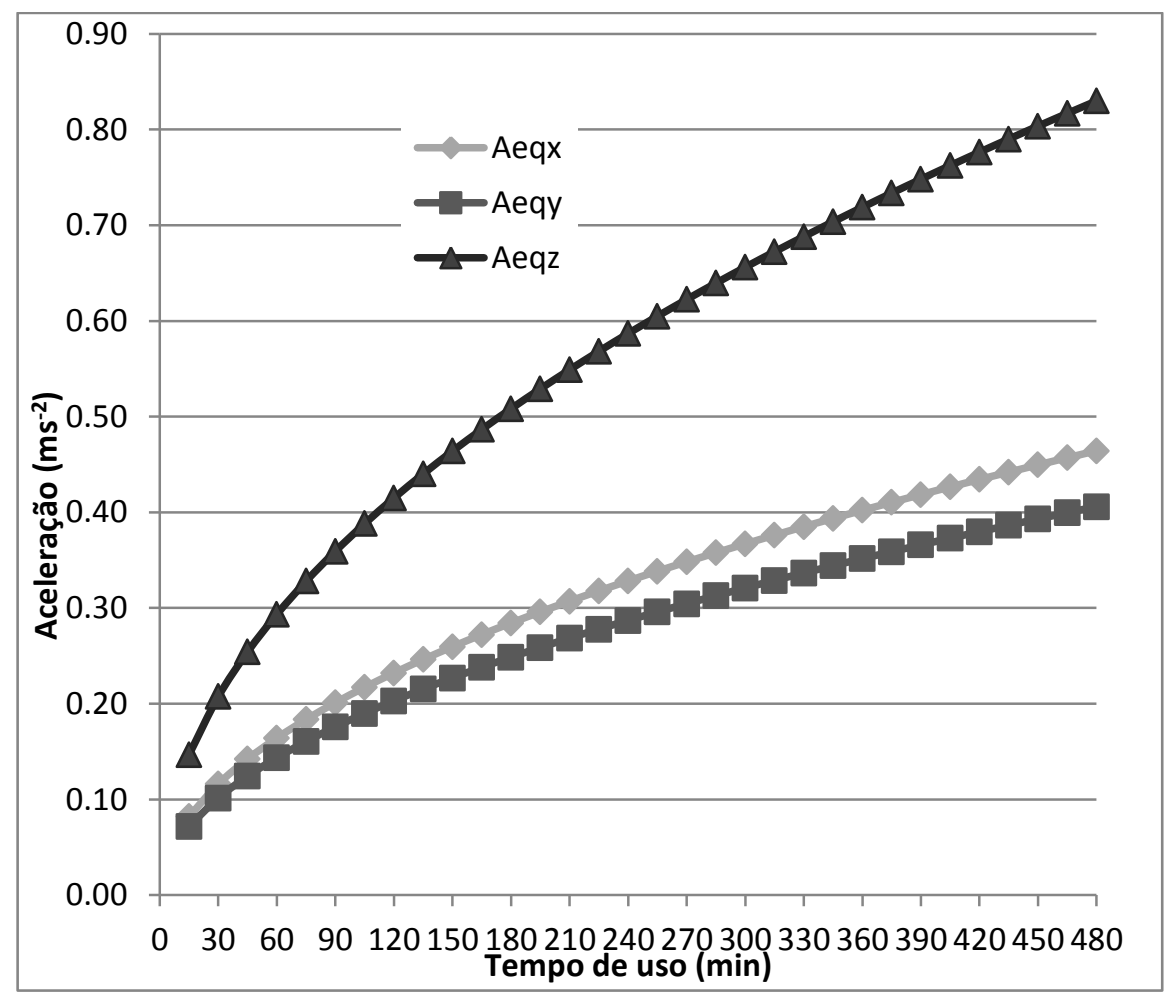

Figura 1 - Exposição do passageiro acomodado em assento central à VCI pelo método básico. Fonte: "com os próprios cálculos".

De acordo com os dados, o passageiro da primeira fila somente atingirá o nível de ação, de 0,5 ms-2, a partir de 375 min de exposição ou $6 \mathrm{~h} 25 \mathrm{~min}$ acomodado no interior do veículo. As observações sugerem que tal situação ocorreria somente para casos excepcionalmente esporádicos e de rara possibilidade já que, mesmo que o passageiro percorra uma linha completa (ida e volta), o tempo não excederia a 1,63h.

0 mesmo procedimento de análise, utilizando o método da quarta-potência, não gerou valores de exposição considerados como risco, do ponto de vista ocupacional, uma vez que todos se encontravam inferiores ao nível de ação de 9,1 ms-1,75. Para o passageiro acomodado na poltrona central do veículo o nível de ação somente seria atingido após 180min ou 3h de exposição. Pelo método da quarta potência, todos os valores estiveram abaixo do nível de ação.

É possível estabelecer, com os dados dos valores de exposição à VCI expostos na Tabela 2, comparação entre os estímulos recebidos durante o trajeto, pelo motorista, cobrador e a passageira.

Tabela 2 - Distribuição dos valores médios das magnitudes de exposição à VCI, de acordo com os dois métodos de avaliação , para motorista, cobrador e passageiro. Fonte: "com os próprios cálculos".

\begin{tabular}{|c|c|c|c|c|}
\hline \multirow{2}{*}{$\begin{array}{l}\text { Magnitudes de } \\
\text { VCI }\end{array}$} & \multicolumn{2}{|c|}{ Condutor } & \multicolumn{2}{|c|}{ Passageiro } \\
\hline & Cobrador & Motorista & $\begin{array}{l}\text { Assento } \\
\text { frontal }\end{array}$ & $\begin{array}{c}\text { Assento } \\
\text { central }\end{array}$ \\
\hline $\operatorname{Aeq}(\mathrm{x})-\mathrm{ms}^{-2}$ & 0,34 & 0,33 & 0,32 & 0,33 \\
\hline $\mathrm{Aeq}(\mathrm{y})-\mathrm{ms}^{-2}$ & 0,45 & 0,32 & 0,35 & 0,29 \\
\hline $\operatorname{Aeq}(\mathrm{z})-\mathrm{ms}^{-1,75}$ & 0,74 & 0,64 & 0,56 & 0,83 \\
\hline $\mathrm{VDVx}-\mathrm{ms}^{-1,75}$ & 2,37 & 2,74 & 3,65 & 2,97 \\
\hline $\mathrm{VDVy}-\mathrm{ms}^{-1,75}$ & 4,00 & 2,58 & 4,12 & 2,47 \\
\hline $\mathrm{VDVz}-\mathrm{ms}^{-1,75}$ & 6,30 & 7,26 & 5,06 & 7,21 \\
\hline
\end{tabular}

Do ponto de vista de conforto do passageiro, verificou-se que, de acordo com o método básico estabelecido na ISO 2631/1 (1997), o assento frontal foi considerado "um pouco desconfortável". Entretanto no assento central, em referência ao eixo z, a classificação foi enquadrada como "desconfortável". Para os demais eixos, neste mesmo ponto, "um pouco desconfortável".

Ao considerar as diferenças registradas entre os assentos do motorista e cobrador, dotados de sistema de amortecimento e o assento rígido do passageiro, constatou-se que os valores médios das magnitudes de exposição são similares o que sugere pouca eficiência de amortecimento da vibração do assento dos condutores.

\section{Conclusão}

Este estudo teve como objetivo avaliar os níveis de exposição à VCI de motorista, cobrador e passageiro assentado em duas seções do veículo. Foi possível constatar que os postos de trabalho dos condutores não apresentam a devida adequação para o amortecimento necessário do estímulo estudado, uma vez que valores observados superam os níveis limites estabelecidos pela normalização. Salienta-se que os assentos destes trabalhadores não apresentam configuração apropriada, pois os valores encontrados são similares aos medidos nos assentos do passageiro, que apresenta uma estrutura rígida. Assim, há um risco importante de ocorrência de dor lombar entre os condutores. No tocante à realidade do 
passageiro, a situação foi classificada como "pouco confortável" e "desconfortável", em especial no eixo z, para o assento na seção central do veículo. No entanto, confrontando com os limites definidos para a ocupação, a exposição do passageiro se manteve abaixo dos níveis de ação. Recomenda-se a adoção de estudos com maior extensão e profundidade, dada a limitação deste estudo, com o fim de contribuir para um transporte público por ônibus com maior conforto e menor risco para os condutores.

\title{
Referências
}

Brasil. (1999). Portaria no 1339/GM, de18 de novembro de 1999. Lista de doenças relacionadas ao trabalho.

Griffin, M. J. (1998). Predicting the hazards of whole body vibration - considerations of a standard. Industrial Health, 36, 83-91.

Griffin, M.J.; Howarth, H.V.C.; Pitts, P.M.; Fischer, S.; Kaulbars, U.; Donati, P.M., \& Brereton, P.F. (2006). Guide to good practice on whole-body vibration. Non-binding guide to good practice with a view to implementation of Directive 2002/44/EC on the minimum health and safety requirements regarding the exposure of workers to the risks arising from physical agents (vibrations). Luxembourg, European Commission.

International Organization for Standardization. ISO 2631: Mechanical vibration and shock - Evaluation of human exposure to whole-body vibration - Part 1: General requirements. Draft International. (1997). Standard. Geneva.

Nelson, C. M., \& Brereton, P. F. (2005). The European Vibration Directive. Industrial Health, 43, 472-749.

Parlamento Europeu; Conselho da União Europeia. (2002). Directiva 2002/44/CE do Parlamento Europeu e do Conselho, de 25 de junho de 2002, relativa às prescrições mínimas de segurança e saúde respeitantes à exposição dos trabalhadores aos riscos devidos aos agentes físicos (vibrações) (décima sexta directiva especial na acepção do no 1 do artigo 16 da Directiva 89/391/CEE). Jornal Oficial da União Europeia, n L 177, 13-19.

Silva, L. F., \& Correia, F.N. (20125). Avaliação da exposição de passageiros ao ruído no interior de ônibus do transporte público do município de Itajubá. Revista CEFAC,14(1), 57-64.

Silva, L. F., \& Mendes, R. (2005). Exposição combinada entre ruído e vibração e seus efeitos sobre a audição de trabalhadores. Revista Saúde Pública, 39, 9-17.

\begin{abstract}
The whole-body vibration (WBV) is present in several vehicles, including city buses. Excessive exposure is associated with the risk of disorders in the lower back. This study aimed to evaluate the exposure of drivers and passengers to the VCI. It was used the ISO-2631-1997 as a reference standard for evaluation, and the occupational limits of European Directive (2002). There was overcoming the level of action, according to the basic method, the three axes for both the collector and to the bus driver. Overcoming the threshold level, second the method of the fourth power, occurred on the $\mathrm{z}$ axis, to collector and driver, with VDV values of $36.28 \mathrm{~ms}-1.75$ and $39.71 \mathrm{~ms}-1.75$, respectively. To the reality of the passenger, the situation in front of the vehicle seat, the observed exposure to VCI, second the basic method was considered "a little uncomfortable", while for sitting in the middle section, was classified as "uncomfortable". The average values of vibration magnitudes, by the basic method, on the $\mathrm{z}$ axis, found on the seats of passengers and drivers are similar, suggesting that the seat cushioning of these is inappropriate. Drivers are exposed to levels above the recommended limits, and passengers have traveled in an uncomfortable situation.
\end{abstract}

Key words: whole-body vibration, passengers, drivers, conductors, comfort. 